\title{
Black Holes in Role, Essence and Function
}

\author{
Wassim S. Daher \\ Beirut, Lebanon \\ Email: daherw@terra.net.lb
}

How to cite this paper: Daher, W.S. (2021) Black Holes in Role, Essence and Function. Journal of Modern Physics, 12, 744-760. https://doi.org/10.4236/jmp.2021.126048

Received: March 18, 2021

Accepted: May 7, 2021

Published: May 10, 2021

Copyright (c) 2021 by author(s) and Scientific Research Publishing Inc. This work is licensed under the Creative Commons Attribution International License (CC BY 4.0).

http://creativecommons.org/licenses/by/4.0/

\begin{abstract}
This study endeavors to explain black holes through their role, essence and function. It utilizes a customized hypothetico-deductive methodology as a cognitive approach to construct and explain the model. The paper presents schematics as illustrations. Black holes are formed through a clash of Universal energy ripples, or through stellar collapse. Its micro level roles are to: recycle cosmological debris, stabilize the formation of galaxies, define the shape of galaxies, and stratify space around them. Their macro role is a subsystem in the preservation of the Universal balance, construct and shape. Black holes are made of two semi-cores of opposite spins. The cores are heterogeneous and made up of structures of dark matter particles. Surrounding the black hole are the process horizon, event horizon, and trap horizon. Primitive cores cannot survive; black holes which collapse to primitive cores decay and vanish. Black holes attract objects via energy fields, where energy tends to accumulate mass for more complex structure development in which more energy colonizes. Photons can either fall on the event horizon or directly cross to the process horizon depending on the black hole structure. This paper has transformed black holes from a set of scattered and vague ideas into structured objects of defined and necessary universal roles. The paper calls for empirical validation or falsification of its model, theoretical model, and hypothesis.
\end{abstract}

\section{Keywords}

Dark Energy, Dark Matter, Energy Ripples, Galaxies, Processing Core

\section{Introduction}

This paper belongs to a series that endeavors to perceive and comprehend the Universe from a novel perspective. It adopts a customized form of hypothetico-deductive approach [1] [2]. There were different perspectives towards the adopted methodology [3] [4]; however, this paper will not venture further into 
the debate [5] [6]. This study assumes a number of theories that make up a hypothetical model of one part of the Universe, the black hole in this case. After the introduction of the model, the theories that build up the model are introduced. Then the hypothesis presents the main ideas on which the whole model relies.

This cognitive paradigm was introduced and discussed in two previous papers [7] [8]. Each paper addresses one aspect of the Universe, as a prerequisite for the following paper. Some of the previously proposed theories may be modified or developed in the process of cognitive presentation as a means of refining the model and not refuting it. The hypothesis is a group of more profound propositions that may call for a revisit of the model should they be falsified.

Objects are primarily identified through their role in the Universe. Their essence remains a secondary aspect necessary for the role. The reason for the existence of any object is its role and contribution to the Universal system. Its existence in its own right is a function of multiple variables that may be related to the local or macro Universal conditions.

Nature is necessarily, according to the cognitive paradigm, an optimizing entity that utilizes its resources of mass and energy efficiently [8]; one can assume multiple objects with multiple roles necessary to preserve this assumption. If, at one location of space, the necessary and essential conditions for the startup of a multiple structures entity, such as a galaxy, exist, then one can assume that debris and failing objects are part of the building process. An optimizing nature necessitates a recycling mechanism as essential for the scheme. Its role is to 'recycle' debris and store or release the constituents, at certain intervals, into space as raw materials for the building process itself. These intervals may depend on thresholds of fluctuations of energy [7] in the vicinity of the black hole. They may also be a function of straying volume of debris, the rate of formation of complex matter, and the strength of the initiating ripple [7] among others. As such, the recycling plant should have adequate ability to attract debris, break it down into primary building materials and release them, ability to release the energy that has been stored in the decomposed structures, and relevant interactive controls.

Another role that is required in the building process is an anchoring structure that acts as a reference for the new forming objects. Due to the huge turbulences created by the Universal dynamics, star dust and formed objects may stray in different directions which may squander the energy and mass involved in the galaxy formation. It is anticipated that planets and stars do change their locations to achieve equilibrium between their buoyancy and other Universal parameters. As such, an anchoring object which regulates the spatial formation of the galaxy is essential in the building process. Any object that, on the macro level, does not have a specific duty, or, on the galactic level, does not fall within the interacting subsystems, may eventually be sucked into a black hole and recycled into raw material.

The formation zones are formed when the ripples initiated by the Universal expansion clash together in a certain locality [7]. This is also coupled by the 
energy tendency to attract masses to increase colonization capacity. This is further discussed below.

The ripples are energy waves transmitted through matter structures that fill the space. When the ripples collide, the released energy integrates dark matter and its structures, in the collision vicinity, into more complex forms of matter [7] [8] until they are perceived as star dust. The energy released by the collision of the ripples dictates the stage of integration of matter. Matter may be integrated into just star dust, or straying objects, or stars and planets in subsystems, or galaxies, or objects and subsystems still undefined to humans. The quantity or degree of integration of the produced objects depends on the quantity of energy dissipated by the collision of the ripples.

Energy colonizes matter. Energy tends to accumulate matter in order to form more complex structures through different energy forms and thus more capacity for energy colonization. For example, matter integration starts with one form of energy till nuclear structures are formed, then with sufficient matter available, nuclear energy forms atoms, then with sufficient matter, more energy forms molecules. So, as energy accumulates matter, energy builds itself complex forms and more complex forms for more energy colonization. This energy can be perceived, for instance, as electrical, nuclear, magnetic, chemical or gravitational. In space, the more the mass, the more the colonizing energy, the greater the attraction of other masses in order to build more complex structures.

In the space vicinity of Earth, the attraction among masses is proportional to certain constants and parameters. In other vicinities of space where different conditions may exist, energy may form different matter structures that can colonize more or less "quantities" of energy. In these parts of space, the attraction forces among masses may be proportional to different constants and parameters.

As the object formation stage ends, and the pull towards the formation vicinity weakens or the matter gets exhausted, the formed objects may stray in space. If there exists no pull towards a center, the formed objects eventually scatter in space. The different density zones decompose and the formed objects may even crash with each other.

As the formation energy depletes, nature ends up with objects floating at different density zones, debris, and different mater formations that failed to float away from the formation area. A certain "Object" has to form, organize, and preserve the different density zones, recycle debris, and maintain equilibrium in the newly formed environment, the galaxy. If this Object does not exist, the galaxy disintegrates. This "Object" is referred to as a 'black hole'.

\section{Literature Review}

A classic definition of a black hole would be: a body with a high gravitational force such that the escape velocity exceeds the speed of light. Black hole can be defined as a "region where space is falling faster than light" [9].

Black holes have been mainly depicted in mathematical models rather than real 
empirical work [10]. Researchers proposed numerous models of black holes. Many of these models were based on the general relativity theory. Penrose calculated, according to relative relativity, a singularity within a black hole [11]. Schwarzschild [12] among others conjectures that non-rotating black holes are perfectly spherical [13] [14] and their size depends on their mass only [15]. Others showed that the shape and size of rotating black holes at a constant velocity, depends on their mass and rate of rotation [16]. Some scientists argue that a black hole has no hair and can be classified via mass, electric charge, and angular momentum [17] others assume an additional scalar field [18] or soft quantum hair [19].

Some scientists believe that the event horizon has a non-decreasing property [10]. Zeldovich and Starobinsky believe that rotating black holes emit particles [10]. Hawking believed that emitting radiation, hawking radiation, is a property of non-rotating black holes as well, from the area outside the event horizon [10]. J. H. Taylor and R. A. Hulse confirmed that moving heavy objects in space, as predicted by the general relativity theory, emit gravitational waves, at the speed of light, which carry energy away from the emitting object. This will cause stars orbiting each other to spiral in towards each other. [20]

Some researchers presume that black holes form by the collapse of stars due to the gravity of their mass [10] [21] where they collapse into a spherical ball of uniform density [22]. Mathur proposed a fuzz ball model of the black hole which eliminates the singularity model [23]. In a string theory interpretation, black holes behave like "ordinary quantum mechanical objects" [24]. Black holes are interpreted as D-Brains in the string theory [24] [25].

\section{The Model}

This section presents black holes in role, function, and essence. It starts with a discussion of the roles and then continues with an assumption of the essence in light of the function.

\subsection{The Role of Black Holes}

Considering the local or micro role of the object, there are three possibilities for the intervals of formation of the black hole. Following the assumption of resource optimization, the formation time should be dictated by the necessity of foundation of the object's role/s. The first possibility is that the black hole is created as the first galactic object. This implies that it is the first object in any galaxy; and, it is necessarily the prerequisite for the formation of the galaxy; or it can form after the formation of other galactic objects; or, it can never form if the energy dissipated in the clash is below a certain threshold.

In the case the black hole is the first object to be formed, and there are still abundant energy and matter, a galaxy follows. The black hole is a concentrated center of mass colonized by enormous amounts of energy. Energy tends to attract energy in order to accumulate mass for further energy colonization. This makes it the destination of any and all energy colonized structures in its vicinity. 
The structures attracted by the energy pull tendency have various densities. More compacted structures are heavier in density and thus form the first "sea" of matter, "sea 1" around the black hole. Less compacted structures, attracted by the black hole energy, but have less density than the more compacted structures form another sea of matter, sea 2 , around sea 1 . This continues until the black hole stratifies the space around it (Figure 1). The newly formed stars and planets float on/in these seas according to their densities. The stars and planets are attracted to the black hole as energy tends to accumulate mass. However, their density forbids them from sinking into the sea they are floating on.

If energy is abundant and matter is not abundant for the formation of other stars or planets, then matter can be compensated from two sources. The first source is the energy that will integrate simpler mass structures into star dust. The second source is the black hole itself through trapping and disintegrating passing by matter into simpler matter to be utilized by the abundant energy to form stars and planets. Until adequate matter accumulates to form cosmological objects, the black hole remains on its own as a trap for straying objects.

If matter is abundant and energy is not abundant, then the black hole disintegrates some of the matter into simpler forms and releases their stored energy. In this case, there should be more and greater seas of matter around the black hole. The released energy may or may not be enough to form planets or stars. Some smaller and lighter structures may be formed, such as rocks or comets, that float to the furthest sea due to their light density. At that far distances, their energy might not be enough to keep them attracted to the black hole; and thus they may stray in space. A time may come when the black hole consumes all matter in its vicinity without the formation of any galaxies. In this case, where matter and energy are scarce, the black hole remains on its own and may eventually collapse into a primitive core as will be discussed hereafter.

If the black hole is not the first structure to form in a high energy location, then the formed planets or stars will scatter around the region of formation and move around the turbulences of matter distribution around that area. If no black hole forms, the planets may move in irregular orbits forming a temporary galaxy and may end up clashing with each other, or straying in space. If a black hole is formed in the process, and the only way being due to the collapse of a star, then,

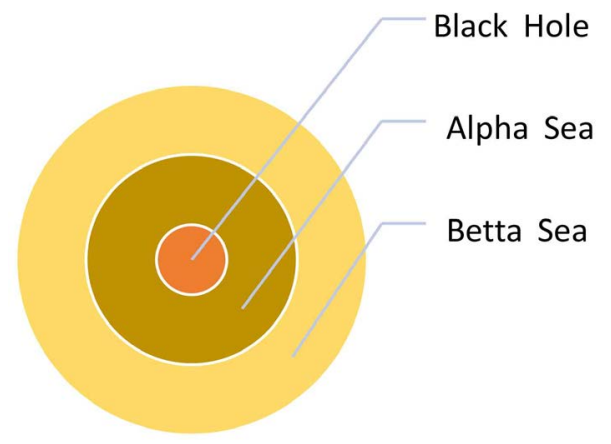

Figure 1. The stratified space around the black hole. 
a galaxy may form and survive. The collapse of a star should be due to a strong concentration of energy within its center. This may be due to an energy ripple, or an energy lump in space that the star passed through, or maybe due to a structure deformation at the time of star formation. The structure of the matter comprising the star may have enormous variation in energy content between the surface and the center of the star which renders it unstable and collapsible at the adequate conditions.

These formed planets and stars in the temporary galaxy are relatively dispersed and gets aligned only after the black hole is created. The black hole stratifies the surrounding space, forming seas of matter, that move the formed objects into their balanced positions in the new forming galaxy. In the process, some objects that are dense enough and fail to float on any of the seas will be sucked in and recycled by the black hole. If the black hole is strong enough, and matter is abundant, then it will create seas that cover wider space. Dense objects, denser than sea 1, may all be sucked in and recycled. Nearby galaxies, falling within the seas of the new black hole, may also be sucked in and repositioned or even recycled. In the case a high energy black hole remains on its own, it will stabilize and reorganize the spatial location it exists in, playing a macro role that will be discussed hereafter.

So black holes have numerous essential and necessary roles in the Universe. Their first role is to recycle spatial debris and reproduce them as valid universal building material. As the black hole sucks and decomposes debris, it then releases the material and excess energy into space. The released material may well be the initial universal building particle, the dark matter. It may also release larger formation of particles. Particles released from black holes are much smaller than atoms. As matter gets decomposed into its simplest forms, energy is released. The released energy can be dark energy or other more complex forms. Tremendous quantities of simple released matter form the densest area, alpha, around the black hole. The more complex particles released from the black hole area floats on the alpha area to form the betta area, and so on. Each formed structure of particles floats on the denser structure of particles. This process goes on till the galactic boundary is defined. Planets and planetary systems formed float among the different particle levels (alpha, betta...) according to their relative densities.

A role other than the creation of galaxies is the definition of galactic shapes. The formation of the density zones defines the shape of the galaxy and the scatter of the planets. Galaxies are defined by the intersection of numerous black hole seas. A black hole distributes matter among seas that build above each other due to various densities of particles. The combination of the black holes dictates the shape of the galaxy through the intersection and interaction of different seas of matter. Planetary systems and their orbits are also defined by this interaction. This logic explains the planar planetary systems such as the solar system, which floats on one of the seas. The sun is a simple form of a black hole that creates seas of particles around it as well for planets to float on. 
Figure 2 depicts the intersection of the spaces formed by two black holes. The resultant can vary from a disc shaped galaxy, to an ovoid, to other shapes depending on the penetration of the relative seas. If the intersection is among the seas of three main black holes then the galactic shapes become more complex. A galaxy, may in time, include several smaller internal black holes formed by collapsing stars. These black holes change the internal shape and the distribution of planets within the galaxy. The solar system itself, with its flat shape, is either floating on one of the seas of a black hole or is formed by the intersection of the seas of two internal black holes inside the Milky Way.

The black hole can exist for its own right if considered from a macro perspective. There can also be a universal essence for the black hole irrespective of the formation of a galaxy. This role is related to the dynamics of the Universe and the control of its turbulences. Controlling the universal turbulences and absorbing the energy and matter fluctuations are necessary to preserve the shape of the Universe and its balances. The conservation of universal shape [7], the egg shape, has to be supported. The universal expansion process has to be controlled in a manner that impedes a permanent mutation of the shape. For its own preservation and persisting expansion, the shape of the Universe has to maintain its elliptic curvature. This subject is beyond the scope of this paper and will be discussed in future research.

\subsection{Energy Fields}

Mass, in its own right, is a "dumb" vehicle colonized by energy. Energy has the affinity to lump mass together, else any formation of the simplest structure would be impossible. Energy can only manifest itself through matter. The attraction among matter is therefore the property of the stored energy. Its strength and reach are proportional to the volume of the colonizing energy.

This is referred to as "gravity" and "gravitational field" as exerted by the mass itself according to both theories of Newton and relativity. The proportionality to mass, according to this paper, is not an accurate approach. The proportionality, instead, is to the energy stored within the mass. The difference between the two

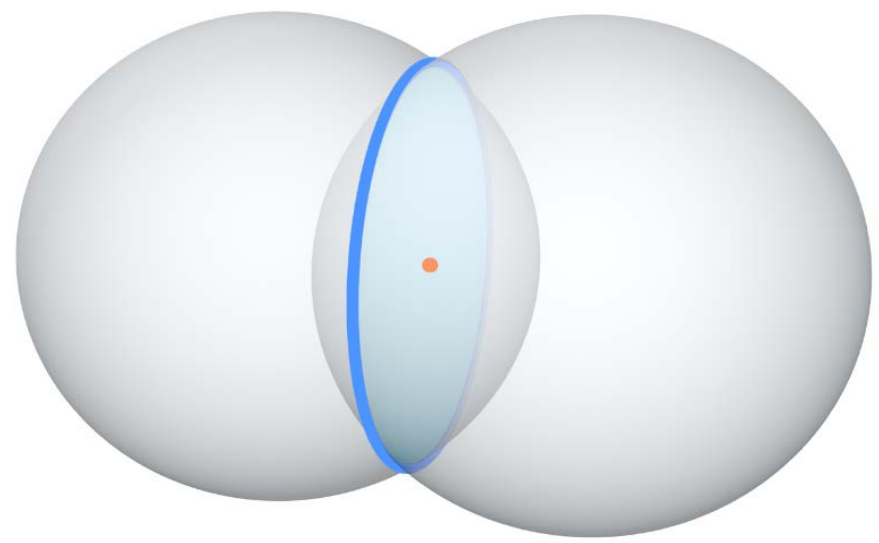

Figure 2. Disc shaped galaxy as an interaction between the seas of two black holes. 
paradigms might not stand out in the present "lump sum" approach of modern science or in the macro scale individualistic perspective of our solar system or even of our galaxy. This, however, may drastically change on micro levels, or in systems, galaxies, or even universes other than ours.

Energy colonizes mass. Energy has the affinity to accumulate. In the process, energy pulls mass together. Energy accumulates mass, to build more complex structures also. More complex structures imply more energy stored. More stored energy implies stronger attraction for more mass; and the cycle goes on.

Energy manifests itself in different forms. Such manifestations are on the nuclear level; others are on the atomic or chemical levels. There are other manifestations of energy on the "pre-nuclear" level, such as what has been referred to in a previous paper [8]. These include the forms of energy on the "dark" level which bridge the states of dark matter and nuclear matter. The forms of energy manifestation will be dealt with in future publications.

\subsection{Primitive Cores}

A primitive core consists solely of dark matter particles. They resemble the state of matter at the end of the compression era [8]. However, a primitive core may not survive if it does not find the means to re-energize itself. Dark matter, in their simplest form and in their own right, cannot store energy. Dark energy builds preliminary structures that encompasses the simplest dark matter particles. These preliminary structures still belong to the dark matter class. As the primitive core consumes its energy, it breaks down these encompassing structures. Its ability to pull the surrounding horizons depletes. The surrounding horizons disintegrate into space. As the encompassing structures are consumed, the core dies and scatters into space.

\subsection{The Core}

A dark matter particle is the simplest particle in our Universe [8]. It has been formed in the compression era of the universe, small " $u$ ", by the integration of the simplest particle and energy into dark matter particles and dark energy. The compression era has created "Universe" in a vast universe and transformed a definite volume of matter into "Matter". Dark matter is the simplest form of Matter in the Universe. Energy conveys itself through Matter. Individual dark matter particles cannot store dark energy in excess of the energy stored upon their formation in the compression era. Dark energy requires building up dark matter particle structures in order to colonize them. The core of black holes consists of dark matter particles in structures that hold weak attraction dark matter particles together. The energy stored in dark matter particles is not enough to keep such particles attracted to each other to form structures or objects.

The energy carried in by the energy ripple waves builds structures of dark matter particles. The amount of energy and matter available in the affected location defines the complexity of these structures as conveyed in Figure 3. 


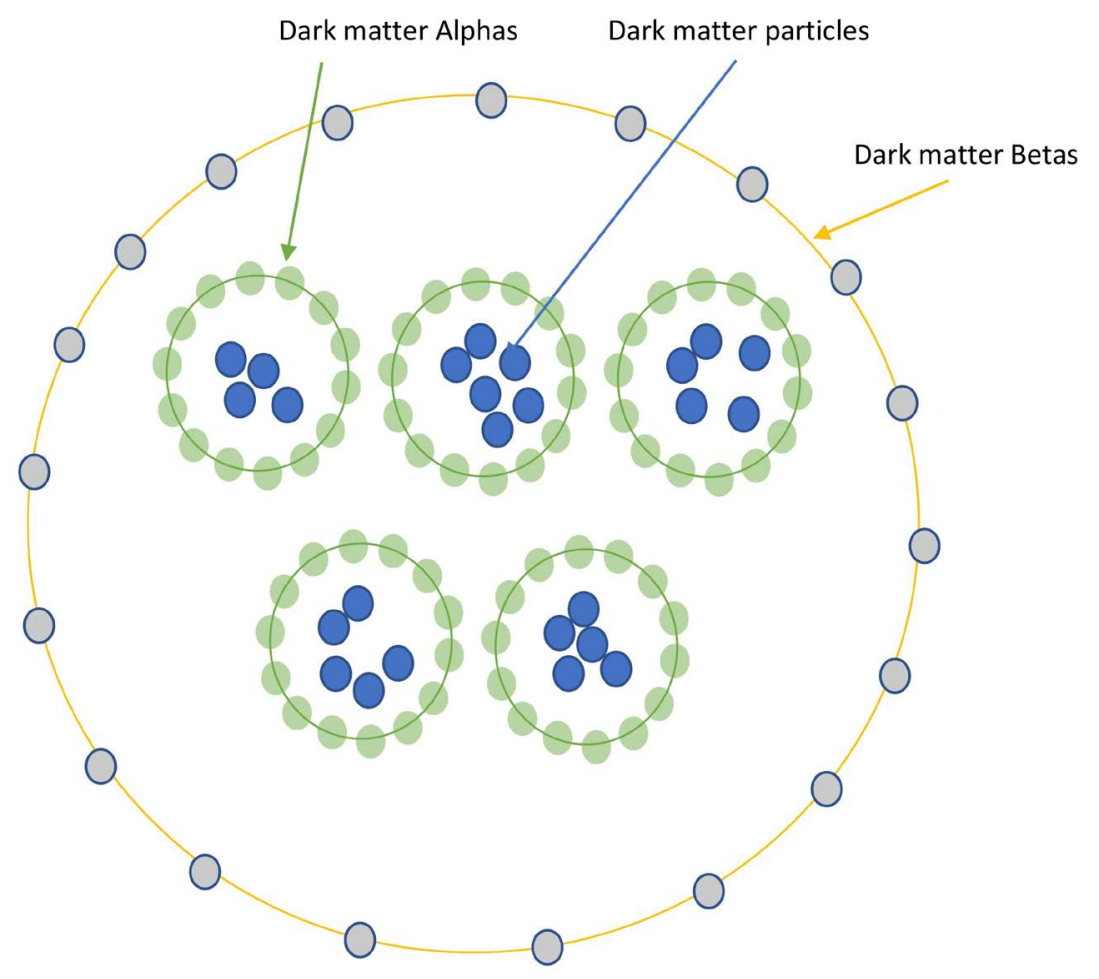

Figure 3. Dark matter structures.

The composition of the processing core is an identity determinant of a black hole. The core consists of a mixture of dark matter particles and simple structures built by these particles. The identity of a black hole varies with the variation of the number of the dark matter particles (Dp) compared to the total number of structured particles (Ds) in the processing core (Dp/Ds). Another defining character is the percentage of each structure in the core per total number of particles (Pt). For alpha particles, for example, it is Dap/Pt. The processing core maintains equilibrium as it continuously processes objects trapped and driven in. The core that consists mainly of alpha particles during equilibrium, will be referred to as "alpha core". As the flow of trapped objects decreases, and the energy in the location is consumed, dark matter particles decompose the Alpha-particles and Dp/Ds increases. As Dp/Ds increases beyond a threshold, the processing core "collapses" into a primitive core, totally consisting of dark matter particles. The composition of the core of the black hole defines the composition of the event horizon. The same applies for a betta-core as it collapses to an alpha core.

Dark energy in the core renders it turbulent, agitating, and rotating. Dark energy integrates dark matter structures that are crushed and formed again. If enough energy is available, the core integrates from primitive to Alpha particle core or beyond, in a process to store energy for future consumption. Amounts of dark energy escape the core via integrated vehicles of dark matter. If the core is not fed with the required amount of energy to maintain its structure, it will collapse from a Betta core to Alpha core or to a primitive core. 
Dark matter particles might have different spins around different axes. In two-dimensions, the spin is either clockwise or anticlockwise. When two particles of the same spin are forced by energy to join, they form a larger structure that preserves the same spin. Momentum is preserved. Other structures of the same spin can join, by energy, to form larger structures with the same spin. Particles of the same spin can join together or repel each other if not able to join. They resemble, more or less, two mechanical gears of the same spin as they approach each other.

At the compression era, when the dark matter particles were formed, part of them had a clockwise spin and the other part a counterclockwise spin, in a two-dimensional perspective. When the expansion era started, and the particles scattered in the Universe, the particles with opposite spins were not distributed equally in all directions. As such, at any given location, one of the spins will prevail. So, at a certain location where the energy ripples hit, and as energy builds dark particles into structures and then objects, the location ends up with two objects of different spins, and with enormous energy colonies, attracting each other, forming the core of the black hole.

The core would be split into two semi-cores with a dominant one (Figure 4). The domination is for the larger semi-core. The resultant spin of the two semi-cores would be the spin of the surrounding material extending beyond the event horizon, into the trap horizon.

As the two semi-cores rotate in opposite directions, around two parallel axes, and attracting each other, they suck in material in their direct vicinity (Figure 5) from one side, process them, and eject part of them from the other side. The broken material structure would be the same as or smaller than the core particles. If the core is a beta, then the material would be broken into beta, alpha, and dark matter particles. Some of the new energy may be sucked into the core upgrading it, from alpha to beta, or will be stored inside some of the structures by upgrading them into alpha or beta, for example.

Some of the new energy ejects, in their matter built vehicles, out of the black hole and into space (Figure 6). The matter vehicles may be structures built from dark matter particles but still smaller than alpha structures in size, for example semi-alpha structures.

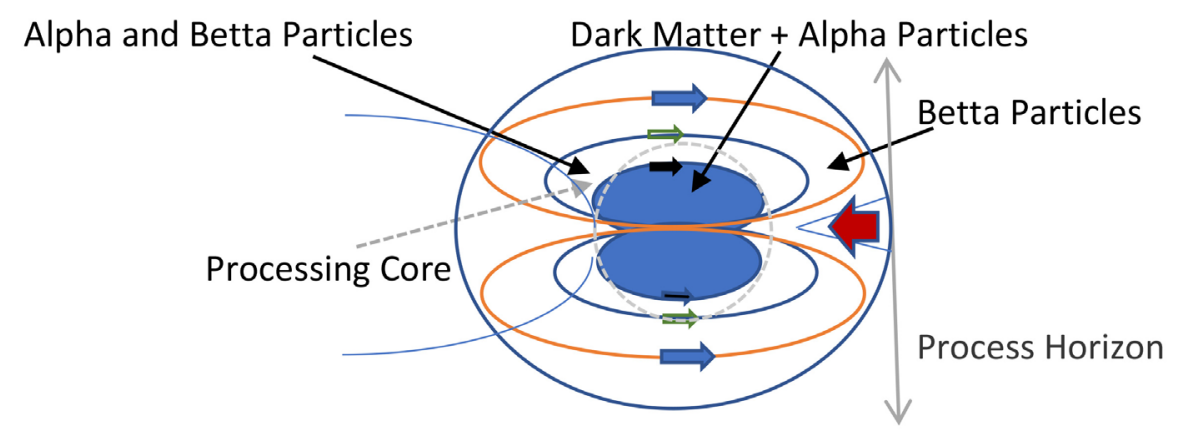

Figure 4. Alpha-core black hole. 


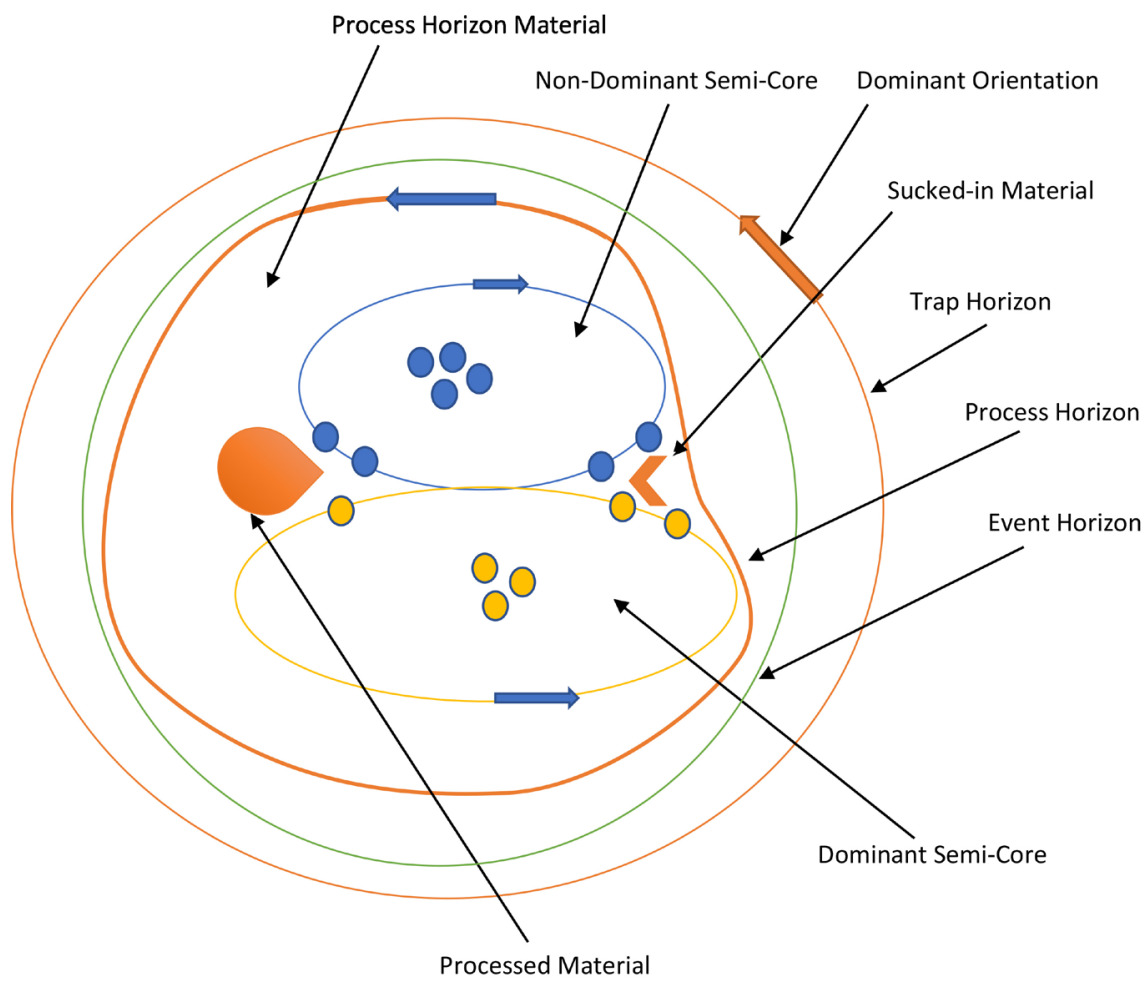

Figure 5. The core of a black hole.

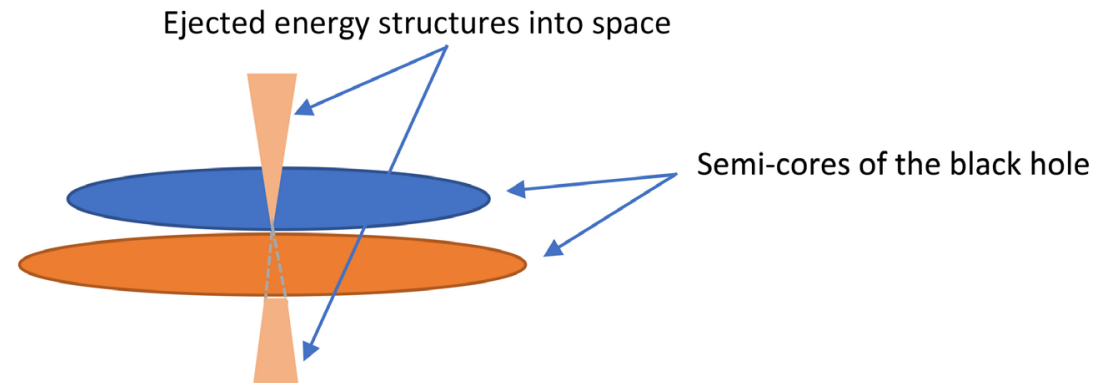

Figure 6. Ejected energy structures from black hole into space.

\subsection{The Space of Horizons}

The space around the black hole in which objects are subjected to direct and severe impact will be referred to as "area of severe impact". This area is composed of three main spaces called "horizons" (Figure 5). The first is the "process horizon" which comprises material in the direct vicinity of the processing core and rotating around it. These materials are sucked in between the two rotating semi-cores to be decomposed and ejected into space or back into the process horizon for further processing. The material of the processing horizon is what falls in through the well-known event horizon.

The event horizon, in this paper, is the furthest space around the black hole that traps photons. Photons are colonized structures that energy uses as vehicles of transportation [8]. As the energy tends to accumulate mass for further struc- 
tural development, photons are attracted to black holes. They penetrate the surrounding horizons until they reach a space comprised of denser structures, a sea of denser structures, on which photons float. That space is called event horizon.

Photons remain rotating in that space till they are broken down by the rotating material into denser structures that can drown further towards the processing horizon. In this case, some energy may escape or get ejected into space in less dense structures than the photons. The event horizon is the "trap horizon" of the photon. The trap horizon is the furthest space around the black hole that traps objects. The trap horizon comprises material orbiting around the black hole. These materials trap and decay passing objects in a particle-blasting effect. The particle-blasted materials sink into the horizons as per their respective densities. The trap horizon radius differs with the densities of the trapped objects. Objects are trapped at different distances from a black hole depending on their particle structures and densities.

For an object to approach a black hole, the buoyancy of the object should be less than force of attraction of the energy. The object, in this case, sinks through the seas of particles towards the black hole. Objects may sink towards black holes in one of several cases. The object may collapse into a heavier object and thus sinks into the sea the object was floating in or on. The object sinks also if the density of the sea the object is floating in or on decreases due to a dying black hole, for example. Another reason for a sinking object toward a black hole may be due to the formation of a new black hole in which a new system of seas is formed.

As the object sinks closer towards the black hole, it reaches a proximity, the trap horizon, where the rotating particles around the black hole particle-blast the object (Figure 7). The blasted material rotates around the event horizon. The particle-blasting process continues until the object is totally decomposed. The decomposed material rotates in the trap horizon where parts of it with densities

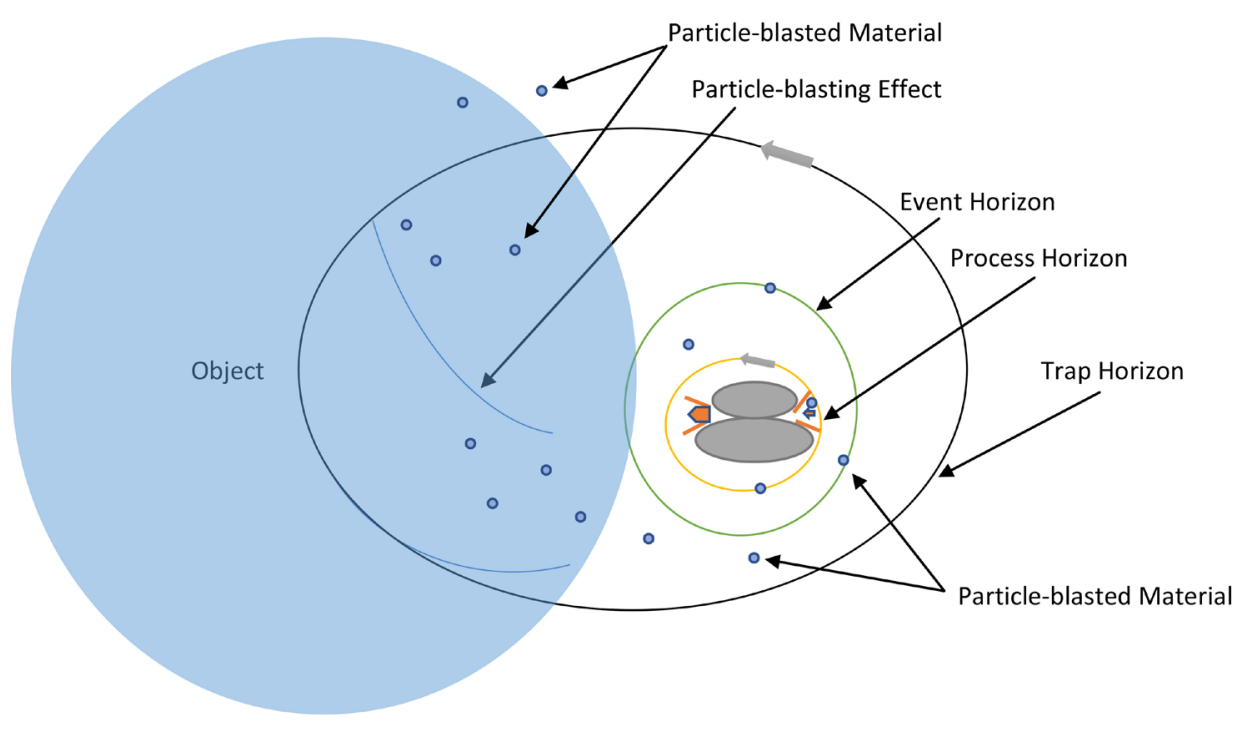

Figure 7. Particle-blasting effect. 
much lower than the density of the trap horizon material may float away into space. Some parts may remain rotating within the trap horizon for long periods becoming part of it. Other parts of the material with high densities may sink to the event horizon or the process horizon and into the processing cores.

\section{Theoretical Model}

1) Energy colonizes matter. Energy accumulates matter forming higher complexity structures through different energy forms and thus greater capacity for energy colonization.

2) Black holes form, organize, and preserve the different density zones, recycle debris, and maintain equilibrium in the newly formed environment, the galaxy.

3) A black hole stratifies the space around it into seas of matter. Matter of similar densities float in the same sea. The seas of matter float over each other as a function of their densities.

4) Black holes are a necessary condition for the formation and survival of galaxies.

5) They define the galactic shape through the formation of the density zones, the seas.

6) The black hole core is split into two semi-cores that rotate in opposite directions.

7) The composition of the processing core and the percentage of each structure in the core per total number of particles $(\mathrm{Pt})$, among others contribute to the identity determinant of a black hole.

8) The area around a black hole is composed of three main spaces called "horizons": The first is the "process horizon", then the "event horizon", then the "trap horizon".

9) The rotational speed and orientation of the process horizon is defined by the resultant in speed and orientation between the two semi-cores.

10) Primitive core cannot survive.

11) Energy fields are created by the affinity of energy to accumulate mass for further structure development. These structures are developed and colonized by more energy.

12) Photons are trapped by some event horizons depending on their size, else they pass to the process horizon. In the event horizon, photons remain until broken down.

13) Trap horizons differ with different objects. The distance that objects are trapped by a black hole varies according to their structures.

\section{Hypothesis}

1) Black holes are a necessary condition for the formation of galaxies.

2) Black holes stratify the surrounding space into seas of matter.

3) Black holes have split cores.

4) The cores consist of structures of black matter. 


\section{Discussion}

This paper presents a complete model that, on one hand, explains numerous findings related to black holes, and on the other hand, refutes other related adopted ideas. In spite of the elusiveness of the subject, nature can only be perceived and thought of as an integrated logic. As such, black holes, and as part of nature, can be depicted through their functions, in a simpler endeavor than to comprehend their essence.

Black holes do not reveal themselves to direct observation but rather through their effect on their surrounding [26]. An example is the supermassive black holes at the centre of galaxies in Active Galactic Nuclei [26] [27]. Such black holes are probably the initial ones created as prerequisites for galaxies, as proposed by this paper. Other smaller black holes are scattered through galaxies [9]; as they give the galaxies their shapes through stratification of seas of matter.

Accepted indications of the presence of black holes are the concentration of masses in small spaces; they are assumed to form via collapsing star cores [10] [27]. As energy tends to accumulate matter for integration into more complex structures; and as the dark matter particles are integrated by energy in the ripple clash vicinity into more complex structures; cores of the black holes are formed of compacted dark matter structures.

When a black hole forms after a collapse of two black holes of different sizes, it initially looks like a chestnut, "with a cusp on one side and a wider, smoother back on the other" [28]. Scientists conjectured that black holes are spherical in shape, if they are non-rotating or form due to a gravitational collapse of a non-rotating star. If the black hole is rotating, then it bulges outward near its equator. [10] The chestnut shape of a black hole is depicted in Figure 5. It includes the processing cores and the processing horizon.

The probable merger of two black holes initiated gravitational waves detected in 2015 with the Laser Interferometer Gravitational Wave Observatory (LIGO) [29]. This occurrence is explained in this paper as the energy tendency to attract and accumulate matter.

Another phenomena explained by this paper are some of the findings of the Event Horizon Telescope (EHT), namely, what is referred to as the "photon nut" where photons "neither escape to infinity nor fall across the event horizon" [30]. These are photons floating on the event horizon until they are broken into denser structures, and thus fall into the process horizon. They can also be transformed into lighter structures that float with the colonizing energy back into space. This paper explains also the bright ring of emission around the black hole [31], to be the sucked-in and ejected material in the process horizon via the semi-cores of the black hole.

\section{Conclusions}

There is a limited comprehension among researchers of black holes, in essence, in role and in function. Present tools and instruments of science have not been 
able to investigate black holes in essence. These illusive objects are only perceived through their assumed effects on their surroundings.

This paper has invested the power of the mind to explore black holes that, till lately, have only been variables in mathematical equations. A detailed model that respects laws of nature while refuting generally accepted theories has been presented. The model satisfies the necessary universal functions required for an optimizing nature. Then, the structure of the object required to perform those necessary functions has been proposed. The conditions and means for the formation of such objects, namely the black holes, have been presented.

The model to perform has to be structured by milestones defined by a theoretical model that states the main essential ideas for the whole model to work. Statements of the theoretical model can be revisited or modified for an enhanced understanding of black holes. The hypothesis presents the founding ideas for the model. Modification of these statements implies a major modification in the model itself. This research philosophy in its own right can be considered as a road map for empirical research.

Most importantly, this paper has transformed black holes from a set of scattered and vague ideas into structured objects of defined and necessary universal roles. Their necessary correlation with observed phenomena has been explained. Galaxies can only form in the presence of black holes. Multiple black holes define the shapes of galaxies. They also distribute formed stars and planets among the seas of matter the black holes form. Black holes are born and may die and decompose. Subtracting black holes from the Universe renders a chaotic and non-sustainable reality.

Scientific capabilities are still too modest to overcome their limitations in the near future. Mathematical equations can only reflect the limitations of the mind to identify the entailed variables. This paper belongs to a series of research that calls for releasing the human mind from materialistic constraints when it comes to understanding the Universe.

The limitations of the study are in the tools to empirically test and verify the proposed model. The validity of the proposed model may be obtained from the coherence of its ideas. There are some relevant data available; however, the interpretation is still speculative.

The human mind is the most powerful tool for discovering the Universe. Human's comprehension of such a complex system is complemented by one's understanding of its integrated subsystems. Empirical scientists are encouraged to confirm or negate the model building ideas in order to refine it further.

\section{Conflicts of Interest}

The author declares no conflicts of interest regarding the publication of this paper.

\section{References}

[1] Smeenk, C. and Ellis, G. (2017) Philosophy of Cosmology. In: Zalta, E.N., Ed., The 
Stanford Encyclopedia of Philosophy (Winter 2017 Edition), The Metaphysics Research Lab, Stanford.

https://plato.stanford.edu/archives/win2017/entries/cosmology

[2] Gale, G. (2019) Cosmology: Methodological Debates in the 1930s and 1940s. In: Zalta, E.N., Ed., The Stanford Encyclopedia of Philosophy (Summer 2019 Edition), The Metaphysics Research Lab, Stanford.

https://plato.stanford.edu/archives/sum2019/entries/cosmology-30s

[3] Milne, E.A. (1934) Philosophy, 9, 19-38. https://doi.org/10.1017/S0031819100030564

[4] Milne, E.A. (1929) The Aims of Mathematical Physics. Oxford University Press, Oxford.

[5] Eddington, A.S. (1939) The Philosophy of Physical Science. Macmillan, New York.

[6] Whittaker, E.T. (1941) Proceedings of the Royal Society (Edinburgh), 61, 160-175. https://doi.org/10.1017/S0080454100006166

[7] Daher, W. (2017) International Journal of Sciences. Basic and Applied Research, 32, 116-133.

[8] Daher, W. (2017) Global Journal of Science Frontier Research, 17, 29.

[9] Hamilton, A.J.S. (2020) General Relativity, Black Holes, and Cosmology. http://timethefinalfrontier.weebly.com/uploads/1/9/7/5/19751121/special relativity and applications.pdf

[10] Hawking, S. (1996) The Illustrated a Brief History of Time. Bantam Books, New York.

[11] Penrose, R. (1965) Physical Review Letters, 14, 57-59.

[12] Schwarzschild, K. (1916) Sitzungsberichte der Königlich Preussischen Akademie der Wissenschaften, 7, 189-196.

[13] Israel, W. (1986) Canadian Journal of Physics, 64, 120-127. https://doi.org/10.1139/p86-018

[14] Israel, W. and Poisson, E. (1990) Physical Review D: Particles and Fields, 41, 17961809. https://doi.org/10.1103/PhysRevD.41.1796

[15] Taylor, E.F. and Wheeler, J.A. (2000) Exploring Black Holes: Introduction to General Relativity. Addison Wesley, Boston.

[16] Kerr, R.P. (1963) Physical Review Letters, 11, 237-238.

https://doi.org/10.1103/PhysRevLett.11.237

[17] Misner, C.W., Thorne, K.S. and Wheeler, J.A. (1973) Gravitation. W. H. Freeman, San Francisco, 875-876.

[18] Zloshchastiev, K.G. (2005) Physical Review Letters, 94, Article ID: 121101. https://doi.org/10.1103/PhysRevLett.94.121101

[19] Hawking, S.W., Perry, M.J. and Strominger, A. (2016) Physical Review Letters, 116, Article ID: 231301. https://doi.org/10.1103/PhysRevLett.116.231301

[20] The Noble Prize (1993) The Noble Prize in 1993: Press Release.

[21] Chandrasekhar, S. (1931) Monthly Notices of the Royal Astronomical Society, 91, 456-466. https://doi.org/10.1093/mnras/91.5.456

[22] Oppenheimer, J.R. and Snyder, H. (1939) Physical Review, 56, 455-459. https://doi.org/10.1103/PhysRev.56.455

[23] Mathur, S.D. (2008) Journal of Computational and Theoretical Nanoscience, 2, No. 2 . 
[24] Maldacena, J. (2011) Black Holes and the Information Paradox in String Theory. https://www.ias.edu/ideas/2011/maldacena-black-holes-string-theory https://doi.org/10.1142/97898143745520004

[25] Polchinski, J. (1995) Physical Review Letters, 75, 4724-4727. https://doi.org/10.1103/PhysRevLett.75.4724

[26] Seyfert, C.K. (1943) The Astrophysical Journal, 97, 28-40. https://doi.org/10.1086/144488

[27] McClintock, J.E. (2011) Measuring the Spins of Accreting Black Holes.

[28] Calderon Bustillo, J., et al. (2020) Communications Physics, 3, 176.

[29] Abbott, et al. (2016) Physical Review Letters, 116, Article ID: 061102. https://doi.org/10.1038/s42005-020-00446-7

[30] Johnson, M.D., et al. (2020) Science Advances, 6, eaaz1310.

[31] Akiyama, K., et al. (2019) The Astrophysical Journal Letters, 875, L1. https://doi.org/10.1126/sciadv.aaz1310 